\title{
Application Programming Interface for a Customer Experience Analysis Tool
}

\author{
George KOPSIAFTIS ${ }^{\text {a, }}{ }^{\text {, Ioannis GEORGOULAS }}{ }^{\text {a }}$, Ioannis RALLIS ${ }^{\text {a }}$ \\ Ioannis MARKOULIDAKIS ${ }^{\mathrm{a}}$, Kostis TZANETTIS ${ }^{\mathrm{b}}$, Michael SFAKIANOS ${ }^{\mathrm{b}}$ and \\ Nikolaos DOULAMIS ${ }^{\mathrm{a}}$ \\ ${ }^{a}$ School of Rural and Surveying Engineering, National Technical University of Athens \\ ${ }^{\mathrm{b}}$ AppArt S.A.
}

\begin{abstract}
This paper analyzes the architecture of an application programming interface (API) developed for a novel customer experience tool. The CX tool aims to monitor the customer satisfaction, based on several experience attributes and metrics, such as the Net Promoter Score. The API aims to create an efficient and userfriendly environment, which allow users to utilize all the available features of the customer experience system, including the exploitation of state-of-the-art machine learning algorithms, the analysis of the data and the graphical representation of the results.
\end{abstract}

Keywords. Customer Experience, Net Promoter Score, Machine Learning

\section{Introduction}

Customer Experience (CX) has been recognized over the years as a key factor for the commercial success of many sectors, including telecommunication operators, banks, tourist enterprises, retail stores, etc. The cornerstones of a CX based strategy are the CX metrics, such as the Net Promoter Score (NPS) which expresses the customer's likelihood to recommend the company's product and/or services [1]. Based on an extended data set from the telecommunication sector, several state-of-the-art Machine Learning (ML) where employed in order to clarify the relation between the NPS and the most significant CX attributes [2], [3]. The ML techniques, along with several graphical representation features were incorporated in an integrated application dedicated to the analysis of the NPS index.

\section{The architecture of the customer experience API tool}

The CX API tool has been developed in Python, using the Flask [4] and FastAPI [5] frameworks. Figure 1 is a schematic representation of the CX system architecture. The end-users are able to communicate with the API using widely used web service protocols and standards. Specifically, a json-based [6] request/respond process was adopted,

\footnotetext{
${ }^{1}$ Corresponding Author; E-mail: gkopsiaf@ survey.ntua.gr
} 
according to the following steps: 1) the end-user selects the CX features he wants to analyze and the corresponding parameters through the user interface (UI) of the application, 2) an input.json file is created in accordance with the user preferences, 3) the CX tool performs the calculations and creates an output.json file, which contains the results and the metadata of the analysis and 4) the results are displayed using the user-friendly environment of the applications' front-end.

The current version of the CX API mainly focuses on the telecommunication industry, providing capabilities for detailed analytics for several crucial features of the sector, such as product attributes, touchpoints, website, call center, etc. Figures 2 and 3 present indicative screenshots of the application's UI. During the following steps of its development, the API will be further extended to the touristic sector, and specifically the analysis of the hotel customer satisfaction. It should be noted that the API conforms to the design principles of the Representational State Transfer (REST) architectural style and, therefore, it is flexible enough to adjust to a plethora of topics with minor alterations.

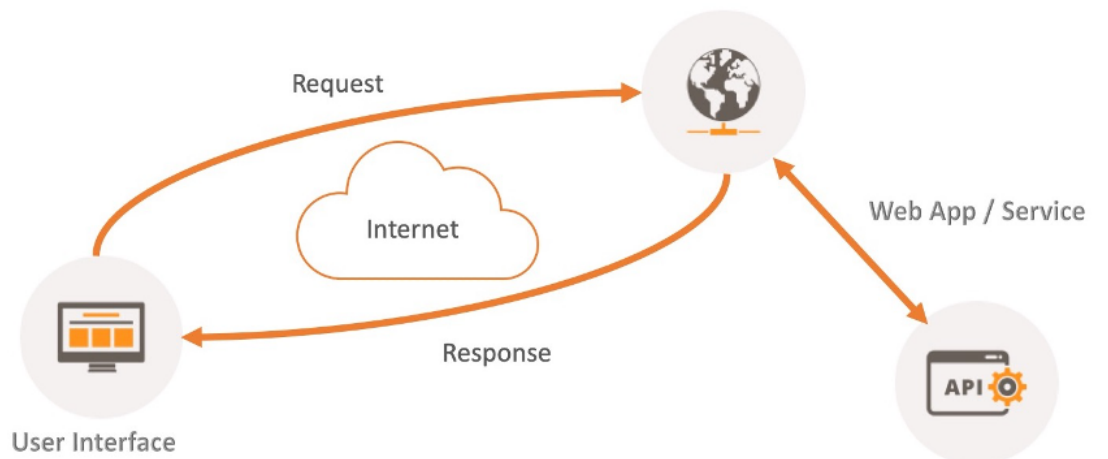

Figure 1. Schematic representation of the CX API tool architecture.

\section{Machine Learning Module}

One of the main objectives of the companies that use CX metrics to form and optimize their commercial strategies is to maximize the NPS through the improvement of the most crucial CX attributes. A major obstacle towards this goal is the lack of an accurate association between the NPS and the CX attribute scores, which, in turns, could be partly attributed to the lack of sufficient data. In order to tackle this deficiency, during an extended research stage, which preceded the development phase of the API, an effort was made to associate the NPS and the CX attribute scores using stat-of-the-art Machine Learning (ML) techniques and specifically the following [2]: 1) Artificial Neural Networks, 2) Convolutional Neural Networks, 3) Support Vector Machines, 3) Linear Regression, 4) Random Forest, 5) Decision Trees, 6) Naive Bayes and 7) K-Nearest Neighbors. All these techniques were calibrated using a relatively limited initial dataset, which 


4. Customer Effort Analysis

Figure 2. Screenshot of the CX API, presenting the features which are available for analysis for the telecommunication sector.
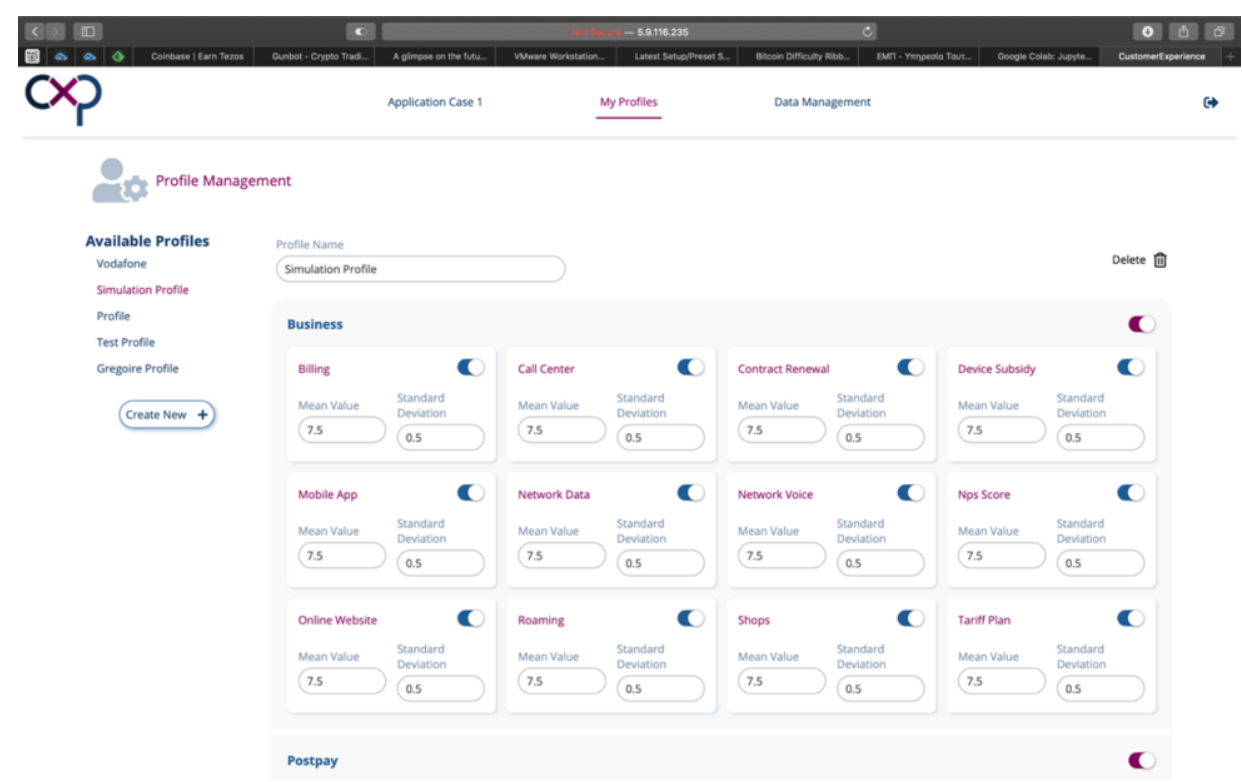

Figure 3. Indicative screenshots of the CX UI, depicting the available options in the Operations Analysis section of the API.

was based on true reviews from telecommunication companies. During the preparatory phase of the API the aforementioned ML techniques were incorporated in a ML module, which was integrated in the final version of the API. The ML module proved to be quite important for the API, as it allowed the formulation of several Use Case Scenarios and the full testing of the API's functionalities. The novelty of the overall CX system could be established on the utilization of advanced ML algorithms to tackle the major obstacles in the evaluation of the CX metrics: 1) the lack of sufficient data and 2) the fact that the CX metric scores depend not only on the CX attributes, but also on other unidentified attributes, such as perception or emotional related attributes. As shown in previous studies [2] the use of the ML module significantly improved most of the examined statistical 
metrics. Further investigation will be performed in the following steps of the CX system development to test the ability of the proposed methods to capture the relation between the CX attributes and the NPS indicator.

\section{Conclusions}

This paper briefly describes a CX application, which has the form of an integrated system, including a novel CX analysis tool, as well as a user-friendly UI. The application is currently at the last stages of its development and a full functional version will be soon available for both research and commercial purposes.

\section{Acknowledgment}

This research has been co-financed by the European Union and Greek national funds through the Operational Program Competitiveness, Entrepreneurship and Innovation, under the call RESEARCH - CREATE - INNOVATE (project code: T1EDK-05063).

\section{References}

[1] F.F. Reichheld, The one number you need to grow, Harvard business review, 81(12), 46-55, 2003.

[2] I. Markoulidakis, I. Rallis, I. Georgoulas, G. Kopsiaftis, A. Doulamis and N. Doulamis, A Machine Learning Based Classification Method for Customer Experience Survey Analysis, Technologies 8, 76, https://doi.org/10.3390/technologies8040076, 2020.

[3] I. Rallis, I. Markoulidakis, I. Georgoulas, and G. Kopsiaftis, G., A novel classification method for customer experience survey analysis, In Proceedings of the 13th ACM International Conference on PErvasive Technologies Related to Assistive Environments (pp. 1-9), 2020.

[4] Flask, 2018, [Online]. Available: https://flask.palletsprojects.com/en/1.1.x/

[5] FastAPI, 2021, [Online], Available: https://fastapi.tiangolo.com/

[6] JSON, 2013, [Online], Available: https://www.json.org/json-en.html 